\title{
Cutaneous Larva Migrans in Urban Area: A Case Report
}

\author{
Tiffany Roelan ${ }^{1}$ \\ ${ }^{1}$ General Practitioner, Kodiklatad Pusdikkes Pratama Clinic, East Jakarta, Indonesia.
}

\begin{abstract}
Cutaneous larva migrans (CLM) is a zoonotic skin disorder caused by the infestation of animal hookworm larvae, the most common being Ancylostoma braziliense. The larvae penetrate the skin surface and subsequently migrate to the epidermis, leaving a typical manifestation of linear or serpiginous erythematous burrow-like tracks, known as creeping eruption. This report presented a case of a young man with complaints of the serpiginous erupted lesion over the upper left thigh, following a recent history of beach travel. On dermatological examination, an erythematous serpiginous lesion and papules were found, portraying the clinical presentation of CLM. The administration of albendazole $400 \mathrm{mg}$ was started, and the symptoms were completely resolved.
\end{abstract}

Keywords: larva migrans, skin disease, creeping eruption, nematodes, albendazole.

\section{INTRODUCTION}

Cutaneous larva migrans (CLM) is an infestation of the skin by penetration and migration of nematode larvae, specifically animal hookworm, in the epidermis layer of the skin. It is generally distributed around tropical and subtropical regions, initiated by direct skin contact to the contaminated soil or sand containing causative parasitic worms. ${ }^{[1,2]}$ Clinically, CLM is characterized by vaguely erythematous raised tracks on the skin, with a major predilection on the exposed skin and extremities. The lesion typically appears as a single or multiple, linear or serpiginous burrow-like tracks, more or less ramified and intertwined. The lesion varies in size, starting from a few centimeters in length and width between 2 to 4 millimeters. Because of the inflammatory reaction resulting from the eruption of larvae in the skin, the manifestation of CLM is often accompanied by pruritus. ${ }^{[3]}$ In this report, we portrayed a case of CLM with a classic presentation on the upper left thigh in an adult patient.

\section{CASE PRESENTATION}

A 21-year-old Indonesian male presented with an intensely pruritic, serpiginous erupted lesion over the upper left thigh. The lesions began prior to the recent history of travelling and laying down on beach sand around the urban area of Jakarta, about a month before the examination. The patient said that the lesion had been slightly increased in length by a few millimeters ever since.

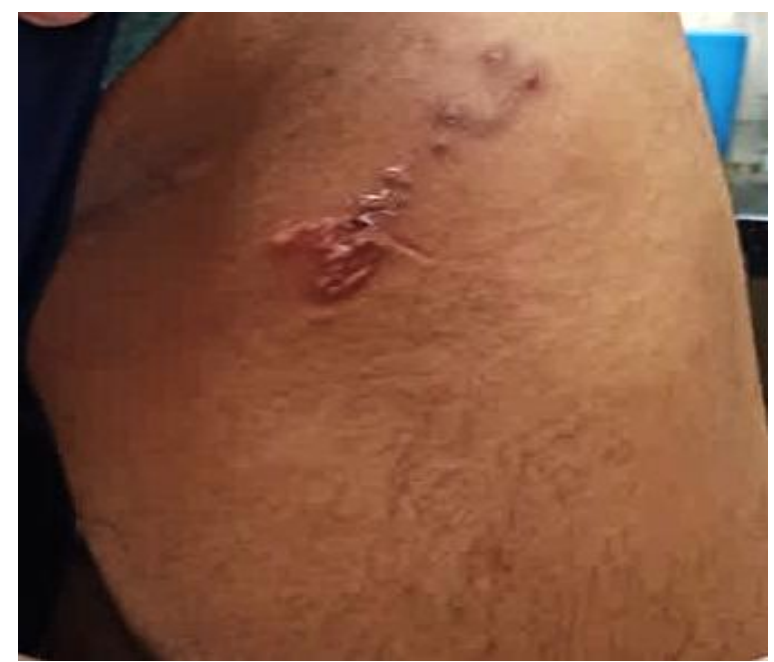

Figure 1. Clinical picture showing the classic serpiginous burrows-like lesion indicating the manifestation of Cutaneous Larva Migrans on the upper left thigh.

The general examination was within the normal limit. Dermatological examination revealed the presence of an 
erythematous, serpiginous skin eruption, surrounded by numerous inflammatory papules, on the upper left thigh (Figure 1). Based on the history and clinical findings, we suspected the patient as a case of CLM.

Histopathological examination was performed and showed an epidermal spongiosis, accompanied by mixed inflammatory infiltrates, mainly eosinophils, in the middle dermis (Figure 2). Additional blood examination showed a high number of circulating eosinophils in the peripheral blood $\left(2.3 \times 10^{9}\right.$ cells/L). Although we did not perform specific examinations, such as enzyme-linked immunosorbent assay (ELISA), the classical finding from the supporting examinations was adequate to confirm the diagnosis of CLM.

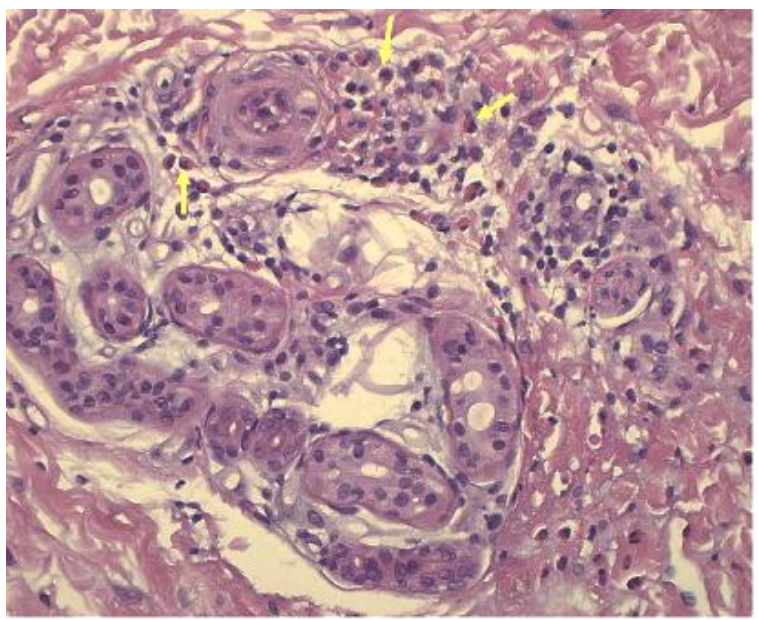

Figure 2. Histopathological examination showing epidermal spongiosis and infiltration of lymphocytes and eosinophils (yellow arrow) in the middle dermis.

The patient was subsequently treated with a course of albendazole (400 mg/day for seven days). In addition, topical corticosteroids and antihistamines were administered for the symptomatic treatment of pruritus. The patient reported significant improvement of symptoms within a few days, and the lesions were completely resolved within one week after treatment.

\section{DISCUSSION}

Cutaneous larva migrans or "creeping eruption" is a distinctive zoonotic skin disorder among travellers returning from vacations in tropical or sub-tropical regions. Various species of parasitic hookworms cause this condition. ${ }^{[4]}$ Animal hookworms are the most common causative agents of CLM among humans, with Ancylostoma braziliense being the most significant species. Less often, CLM is also caused by A. caninum, A. ceylanicum, A. tubaeforme, Uncinaria stenocephala, and Bunostomum phlebotomum. ${ }^{[5]}$

The medical history and clinical findings from the patient thoroughly support to establish the diagnosis of CLM. Initial infection begins with a history of travelling to endemic regions, generally to coastal areas, sandy beaches, or soilage, where the individual spends time walking barefoot. Direct exposure to soil, sand, or feces containing infectious larvae to the exposed skin areas, causes the penetration of larvae into the skin. The larvae will settle in the epidermal layer, particularly between the stratum germinativum and stratum corneum; thus, eventually causing the eruptive appearance of the skin surface in a serpiginous pattern, with slightly increasing length over time. Comparin et al. stated that the diagnosis of CLM is generally sufficient based on the patient's history and clinical findings. However, additional examinations can optionally be performed, such as histopathology, blood counts, and biopsy. ${ }^{[6]}$

Cutaneous larva migrans are often misdiagnosed and treated inappropriately, especially in non-tropical countries, which the incidence is epidemiologically rare. It has to be differentiated from scabies, erythema chronicum migrans, allergic contact dermatitis, and dermatophyte infection. ${ }^{[7]}$ In tropical and developing countries such as Indonesia, the diagnosis of CLM is often confused with scabies skin infection because they both present with burrow-like lesions. Distinguishing the two is not always easy, especially if the latter presents with atypical efflorescence on the upper or lower extremities. Important diagnostic criteria for scabies are the following: a history of exposure to surroundings possibly infested by the mite Sarcoptes scabiei; the presence of crustaceous lesions, papules, generalized 
itching, and rash; and microscopic demonstration of the mite, its eggs, or its feces in skin scrapings. One of the remarkable differences of a scabies infection is the predilection area dominated under the thin body skin, such as the axilla, groin, or interdigital folds of the hand. In cases of CLM, the appearance of single migrating tracts and vesicular or papules lesions commonly involve the lower extremities rather than the hands. Rapid cessation of symptoms after the use of antihelminthic regimens is also an important diagnostic clue. ${ }^{[8]}$

The pathogenesis of CLM involves the role of larvae that is assumed to lack in the collagenase enzymes required to penetrate the human basement membrane; thus, it limits the parasite from invading the dermis. Since humans are not the natural hosts of larvae development, the disease course of CLM is commonly self-limited. Larvae that enter the body are able to persist for only 2-4 weeks, then the resolution of the disease can occur in $80 \%$ of cases. ${ }^{[9]}$ Therefore, CLM generally heals spontaneously within a few weeks or months. The administration of antihelminthic regiments, such as albendazole, thiabendazole, or ivermectin, can shorten the duration of the disease and helps to diminish parasitic agents. The optimal dosage depends on the main causative agent and disease severity. The previous recommendation suggests using albendazole $400 \mathrm{mg}$ oral for three days or a single dose of ivermectin $200 \mathrm{mg} .{ }^{[10,11]}$ Prickett et al. recommend oral ivermectin $(150-200 \mu \mathrm{g} / \mathrm{kg}$ body weight) single dose or oral albendazole (400-800 mg/day) single dose for three days as the first-line therapy for CLM, with cure rates ranging from 94 to 100 percent. The alternative treatments include topical tiabendazole and topical albendazole, which is applied twice a day for ten days (although the availability is still limited in some countries). Invasive methods, such as cryotherapy or frozen surgery using liquid nitrogen and ethyl chloride, are no longer recommended. ${ }^{[12]}$
The presented case responded very well to oral albendazole and topical corticosteroid with antihistamine treatment. These additional symptomatic mediations are important as they help rapidly improve pruritus and total clearance of skin lesions in weeks. No side effect was observed during the treatment period. The publication of this clinical case report is significant to highlight its incidence in an urban area. Healthcare providers should be more attentive to the possibility of CLM, even in urban areas, especially in patients who have a typical clinical picture with a history that supports the source of CLM infection.

\section{CONCLUSION}

Cutaneous larva migrans may occur in urban areas, even among locals with a travel history to beach destinations in tropical and subtropical regions. The establishment of diagnosis is essentially clinical based on the medical history and clinical presentation. The treatments with albendazole provide an excellent agent of choice for a complete resolution of the disease.

\section{Acknowledgement: None}

\section{Conflict of Interest: None}

\section{Source of Funding: None}

\section{REFERENCES}

1. Sow D, Soro F, Javelle E, Simon F, Parola P, Gautret P. Epidemiological profile of cutaneous larva migrans in travelers returning to France between 2003 and 2015. Travel Med Infect Dis. 2017;20:61-4.

2. Heukelbach J, Feldmeier $\mathrm{H}$. Epidemiological and clinical characteristics of hookworm related cutaneous larva migrans. Lancet Infect Dis. 2008;8(5):3029.

3. Blackwell V, Vega-Lopez F. Cutaneous larva migrans: clinical features and management of 44 cases presenting in the returning traveller. $\mathrm{Br} \mathrm{J}$ Dermatol. 2001;145:434-7

4. Bachmeyer C, Moreno-Sabater A. Vesiculobullous cutaneous larva migrans in 
a 29-year-old man, diagnosed using teledermatology. Can Med Assoc J. 2018;190:E888.

5. Kahn CM, Line S, editors. The Merck Veterinary Manual. 10th ed. Whitehouse Station, NJ: Merck and Co; 2010. p.2791-7.

6. Comparin C, Rodrigues MM, Santos BC. Extensive cutaneous larva migrans with eczematous reaction on atypical localization. Am J Trop Med Hyg. 2016; 96(6):1185-6.

7. Bava J, Gongalez LG, Seley CM, Lopez GP, Troncoso A. A case report of cutaneous larva migrans in Argentina. Asian Pac J Trop Biomed. 2011;1:81-2.

8. Otranto D, Eberhard ML. Zoonotic helminths affecting the human eye. Parasit Vectors. 2011;4:41.
9. Veraldi S, Arancio L, 2006. Giant bullous cutaneous larva migrans. Clin Exp Dermatol. 2006;31(4):613-4.

10. Caumes E. Treatment of cutaneous larva migrans. Clin Infect Dis. 2000;30(5):811-4.

11. Wang $\mathrm{S}, \mathrm{Xu} \mathrm{W}, \mathrm{Li} \mathrm{LF}$. Cutaneous larva migrans associated with Löffler's syndrome in a 6-year-old boy. Pediatr Infect Dis J. 2017;36(9):912-4.

12. Prickett KA, Ferringer TC. What's eating you? Cutaneous larva migrans. Cutis 2015; 95:126-8.

How to cite this article: Roelan T. Cutaneous larva migrans in urban area: a case report. International Journal of Research and Review. 2021; 8(9): 224-227. DOI: https://doi.org/10. 52403/ijrr.20210930 